\title{
The Effect of Therapeutic Hypothermia after Cardiac Arrest on the Neurological Outcome and Survival-A Systematic Review of RCTs Published between 2016 and 2020
}

\author{
Christian Colls Garrido ${ }^{1,+}+\left(\mathbb{D}\right.$, Blanca Riquelme Gallego ${ }^{2,3, *,+}+$, Juan Carlos Sánchez García ${ }^{2,4}(\mathbb{D}$, \\ Jonathan Cortés Martín 2,4 (D), María Montiel Troya ${ }^{4,5}$ and Raquel Rodríguez Blanque ${ }^{4,6}$ (D)
}

Citation: Colls Garrido, C.; Riquelme Gallego, B.; Sánchez García, J.C.; Cortés Martín, J.; Montiel Troya, M.; Rodríguez Blanque, R. The Effect of Therapeutic Hypothermia after Cardiac Arrest on the Neurological Outcome and Survival-A Systematic Review of RCTs Published between 2016 and 2020. Int. J. Environ. Res. Public Health 2021, 18, 11817. https:// doi.org/10.3390/ijerph182211817

Academic Editors: Peter Paal,

Hermann Brugger, Mathieu Pasquier and Tomasz Darocha

Received: 13 October 2021

Accepted: 7 November 2021

Published: 11 November 2021

Publisher's Note: MDPI stays neutral with regard to jurisdictional claims in published maps and institutional affiliations.

Copyright: (c) 2021 by the authors. Licensee MDPI, Basel, Switzerland. This article is an open access article distributed under the terms and conditions of the Creative Commons Attribution (CC BY) license (https:/ / creativecommons.org/licenses/by/ $4.0 /)$.
1 Intensive Care Unit, Hospital Universitario Clínico San Cecilio, 18071 Granada, Spain; christian.16.ef@gmail.com

2 School of Nursing, Faculty of Health Sciences, University of Granada, 18071 Granada, Spain; jsangar@ugr.es (J.C.S.G.); jonathan.cortes.martin@gmail.com (J.C.M.)

3 Instituto de Investigación Biosanitaria, ibs.GRANADA, 18012 Granada, Spain

4 Research Group CTS1068, Andalusia Research Plan, Junta de Andalucía, 18014 Granada, Spain; mariamontiel@ugr.es (M.M.T.); raquel.rodriguez.blanque.sspa@juntadeandalucia.es (R.R.B.)

5 School of Nursing Ceuta Campus, Faculty of Health Sciences, University of Granada, 51001 Ceuta, Spain

6 Distrito Sanitario Granada-Metropolitano, 18013 Granada, Spain

* Correspondence: briquel@ugr.es

+ These authors contributed equally to this work.

Abstract: Therapeutic hypothermia is a treatment used for patients who have suffered cardiorespiratory arrest and remain conscious after the recovery of spontaneous circulation. However, its effectiveness is controversial. The objective of this systematic review is to summarize the scientific evidence available about the effect of therapeutic hypothermia on neurological status and survival in this type of patients. Methodology: A primary search in CINAHL, CUIDEN, Pubmed, Web of Science, and Scopus databases was carried out. Randomized clinical trials (RCT) published from 2016 to 2020 were selected. Results: 17 studies were selected for inclusion and most relevant data were extracted. Methodological quality was assessed by the RoB tool. Conclusions: Although therapeutic hypothermia is a safe technique with few adverse and manageable effects, it has not shown to improve survival rate and neurological status of adult nor pediatric patients. It is possible that its positive effect on neuroprotection could be achieved only by preventing hyperthermia although further investigation is needed.

Keywords: induced hypothermia; cardiac arrest; survival rate; critical care

\section{Introduction}

Cardiorespiratory arrest (CA) is one of the main causes of death in developed and developing countries. CA is defined as the cardiac failure caused by the cessation of mechanic activity, which is confirmed by poor circulation. The cardiopulmonary resuscitation (CPR) procedure aims to restore circulation by ensuring ventilation and gas exchange through CPR and defibrillation, among other emergency techniques [1].

Worldwide out-of-hospital cardiorespiratory arrest (OHCA) rate is 83.7 cases per 100,000 per year. In adults, the incidence increases to 95.9 cases per 100,000 annually. These numbers vary between countries and are higher in North America (98.1) or Australia (112.9) in comparison with Asia (52.5). Annually, 300,000 OHCA take place in North America and 275,000 approximately in Europe. Most of them occur at home (66\%), followed by workplaces and public spaces $(20 \%)$. The average age of patients that suffer a CA is 71 years, being more frequent among men than women $[2,3]$.

The main causes of CA are cardiopulmonary (43\%), chronic pulmonary affection (13\%), diabetes (13\%), and expected death (5\%). In $80.3 \%$ of cases, asystole is present, while signs 
of ventricular tachycardia or ventricular fibrillation and electromechanical dissociation are less common (5.9\% and $6 \%$, respectively) [4]. Only $22.8 \%$ of all patients who suffer a CA manage to recover spontaneous circulation. The survival rate of these patients is $4.9 \%$ at 30 days, which increases to $10.4 \%$ when resuscitation is initiated immediately. If CA takes place in a hospital environment, these rates increase to $22 \%$, and mortality prevalence for patients that have recovered spontaneous circulation after a CA and are admitted to hospital is $57 \%$ [5].

In this context, post-resuscitation care after spontaneous circulation recovery becomes vital due to the associated high intra-hospital mortality [6].

Post-resuscitation care aims to control the problem behind the CA, reducing the damage caused by post-CA syndrome. It focuses on neuroprotection in order to prevent further neurological damage after the arrest [7]. More than 50\% of these patients show a sub-optimal neurological status at discharge [8,9]. One of the interventions that appears to be effective to prevent further brain damage is targeted temperature management (TTM), previously known as therapeutic hypothermia (TH) $[10,11]$. It consists on the application of cold through different devices that reduce the patient's temperature in a controlled and gradual manner [12]. It decreases brain metabolism by reducing oxygen and glucose consumption by brain cells [13]. Thus, it modulates the inflammatory response induced during the reperfusion stage by stabilizing enzymatic reactions. In this phase, reactive oxygen species and neurotransmitters are released, generating excitotoxicity [14], so TTM decreases intracranial pressure by enzyme stabilization and vasoconstriction [12]. It is currently also used in cases of anoxic brain injuries, mild brain injuries, stroke, hypoxic ischemic encephalopathy, bacterial meningitis, post-surgical tachycardia, and acute respiratory distress syndrome [15]. When a CA is ongoing, the therapeutic decision to apply TTM must be taken quickly and promptly since it has been shown that its beneficial effects are time dependent. On the other hand, TTM can be applied safely in combination with other interventions for CA, such as percutaneous revascularization [16]. The main objective of this systematic review is to summarize the scientific evidence about TTM effectiveness on neurological status and survival rate in patients who have suffered CA.

\section{Materials and Methods}

A review of TTM studies was carried out to address our scientific question according to Preferred Reporting Items for Systematic reviews and Meta-Analyses (PRISMA). The protocol is registered in PROSPERO coded as CRD42020207405 and available at https: / / www.crd.york.ac.uk/prospero/display_record.php?ID=CRD42020207405 (accessed on 6 November 2021).

Randomized clinical trials (RCTs) relating to TTM and temperature control interventions in patients who suffer a CA and its influence on survival and neurological status were included.

CINAHL, CUIDEN, Scopus, Cochrane, Pubmed, and Web of Science databases and Dialnet journal were consulted. The search was limited to articles written in English or Spanish, and only articles published in the last 5 years were included.

The following MeSH (Medical subject Headings) terms were used to conduct the search strategy: "hypothermia, induced", "induced hypothermia", "heart Arrest", "cardiac arrest", "survival", "survival rate", "survival analysis", "mortality". In addition, some synonyms were used: "therapeutic hypothermia", "induced mild hypothermia", "targeted temperature management", "cardiac arrest", "cardiopulmonary arrest", "cardiorespiratory arrest", and "neurolo". The Boolean operators "AND" and "OR" were used. Therefore, the complete search strategy with the Boolean operators was (("therapeutic hypothermia" OR "hypothermia, induced" OR "induced mild hypothermia" OR "targeted temperature management") AND ("cardiac arrest" OR "heart arrest" OR “cardiopulmonary arrest") AND ("survival" OR "Survival rate" OR "mortality" OR "survival analysis" OR "neurolo *")) OR (("therapeutic hypothermia" OR “induced hypothermia" OR "mild induced hypothermia") 
AND ("cardiac arrest" OR "cardiorespiratory arrest") AND ("survival" OR "survival rate" OR "mortality" OR "survival analysis" OR "neurolo *")).

The inclusion criteria were: RCTs carried out in patients treated with TTM after CA published in the last 5 years (1 January 2016 to 18 April 2020), written in English or Spanish. Animal studies, ongoing RCTs, unavailable full text, TTM studies that did not focus on survival rate and neurological status in patients that suffered CA, systematic reviews, meta-analysis, protocols, and observational and descriptive studies were excluded.

Our electronic search identified 606 potentially relevant studies. Once duplicates were removed $(\mathrm{N}=260), 211$ studies were selected as potentially relevant and reviewed by title and abstract. Seventeen studies were finally included according to the inclusion criteria for full reading and data extraction [17] (Figure 1).

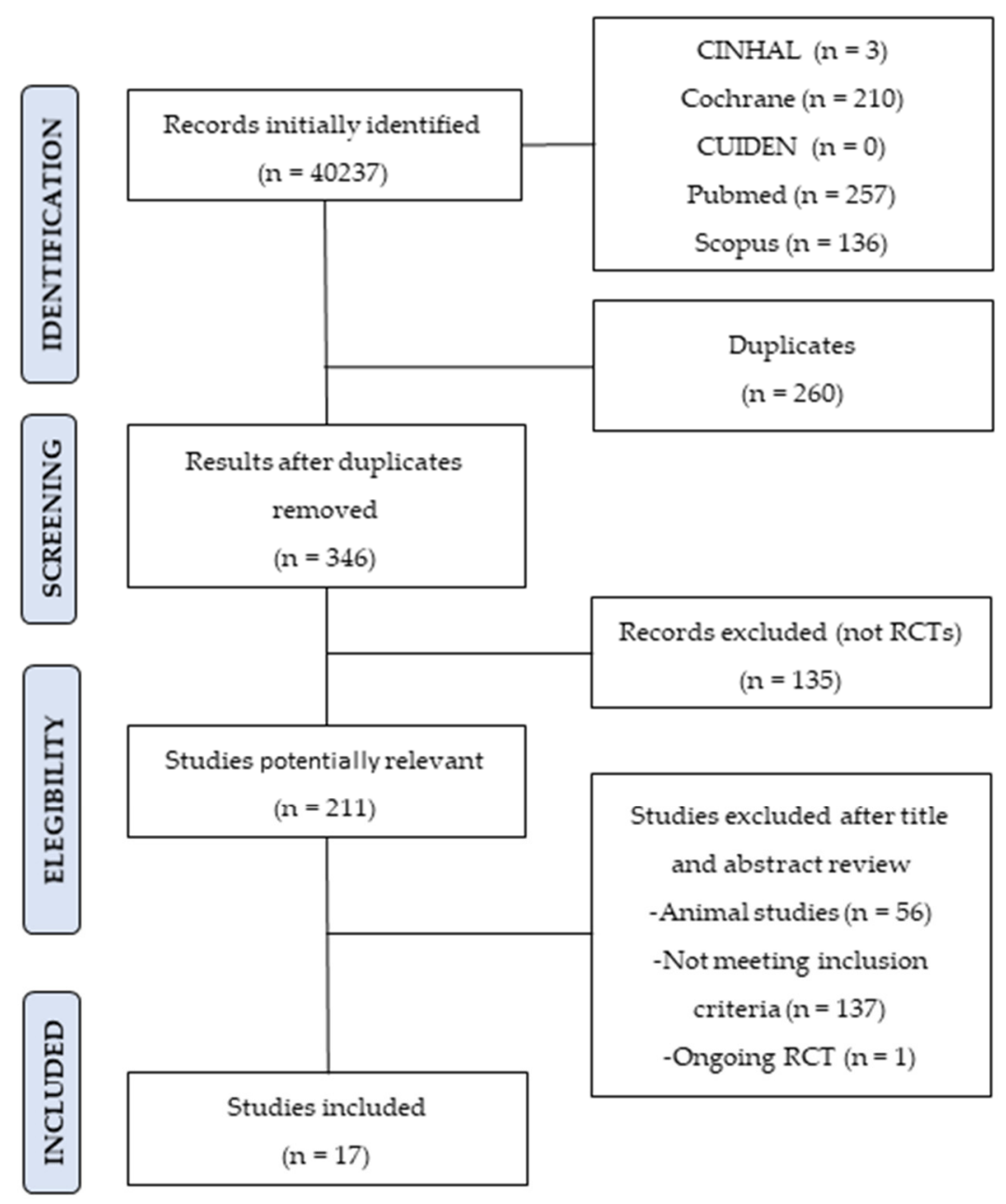

Figure 1. Selection flow diagram.

In order to assess the methodological quality of the selected studies, we assessed the risk of bias in all included studies in duplicate by two independent reviewers (B.R.G. and J.C.S.G.) using the Cochrane risk of bias assessment tool. The following items were assessed: randomization and sequence generation, allocation concealment, blinding and performance, outcome assessment, completeness of outcome data, and selective outcome reporting. Unblinded studies were not penalized in the risk of bias assessment due to the nature of the treatments that makes blinding non-feasible [18].

The information was collected and divided into two groups: adult and pediatric patients. Studies were examined in depth, and the most relevant data were extracted: title, authorship, year of publication, sample size, main objective, treatment in intervention, and control groups and outcomes. 


\section{Results}

In total, 17 randomized trials reporting on 5813 adults and 712 children were included; Tables 1 and 2 show the characteristics of the RCTs carried out in adult and pediatric patients, respectively. Tables 3 and 4 summarize the main results in adult and pediatric patients, respectively. The temperature used in the most frequent experimental group was $33^{\circ} \mathrm{C}$ except in the study of Pang et al. (2016), Scales et al. (2017), and Look et al. (2018), which applied an experimental temperature of $32^{\circ}$ and $34^{\circ}$, respectively. The neurological outcome was assessed though the Cerebral Performance Category scale (CPC) in the majority of trials conducted in adult population and though Vineland Adaptive Behavior Scales (VABS-II) in the pediatric population. The most commonly applied intervention was the combination of invasive and external cooling in adult patients with the exception of Look et al.'s (2018) and Nordberg et al.'s (2019) studies, which only used external cooling. The time after which the survival rate was analyzed was very heterogeneous. It was determined after hospital discharge in three studies Bernard et al. [18], Look et al. [19] and Scales et al. [20] after one month of follow-up in Meert et al. [21], after three months of follow-up in Lascarrou et al. [22], Lopez-de-Sa et al. [23] and Nordberg et al. [24], after six months of follow-up in Cronberg et al. [25], Kirkegaard et al. [26], Lilja et al. [27], Pang et al. [28] and Fink et al. [29], and after one year after suffering CA in Maynard et al. [30], Moler et al. [31] Moler et al. [32], Silverstein et al. [33]. The quality of included studies was overall moderate, with some studies demonstrating a high risk of bias (Figure 2). One study had a high risk of bias for randomization, and $10(10 / 17,58.82 \%)$ had some concerns. Two studies had a high risk of bias for randomization, and six had some concerns. One study had a high risk of bias for allocation concealment. Outcomes assessment (i.e., attrition) was judged to have a high risk of bias in two studies and was inadequate in three studies but good in 12 studies (12/17 70.58\%). Thirteen studies had a low risk of bias for detection (i.e., selective reporting), and 10 had some concerns in outcomes reporting (i.e., incomplete data). 
Table 1. Characteristic of the selected RCTs carried on in adult patients.

\begin{tabular}{|c|c|c|c|c|c|c|c|c|c|}
\hline $\begin{array}{l}\text { Author } \\
\text { (Year) }\end{array}$ & $\mathbf{N}$ & $\begin{array}{c}\text { Baseline } \\
\text { Characteristics }\end{array}$ & $\begin{array}{l}\text { Cooling } \\
\text { System }\end{array}$ & $\begin{array}{c}\text { Mean Time } \\
\text { Reaching } \\
\text { Target Temperature }\end{array}$ & $\begin{array}{c}\text { Target } \\
\text { Temperature }\end{array}$ & $\begin{array}{c}\text { Inclusion } \\
\text { Criteria }\end{array}$ & $\begin{array}{l}\text { Exclusion } \\
\text { Criteria }\end{array}$ & $\begin{array}{c}\text { Time of } \\
\text { Evaluat- } \\
\text { ing } \\
\text { Survival }\end{array}$ & $\begin{array}{c}\text { Time of } \\
\text { Evaluating } \\
\text { Neurological } \\
\text { Outcome }\end{array}$ \\
\hline $\begin{array}{l}\text { Bernard } \\
\text { et al. } \\
\text { (2016) } \\
{[19]}\end{array}$ & 1198 & $\begin{array}{l}\geq 18 \text { years, IV access, and } \\
\text { were still in CA after CPR }\end{array}$ & $\begin{array}{l}\text { Rapid IV infusion of } \\
\text { large-volume }(30 \mathrm{~mL} / \mathrm{kg}), \\
\text { cold saline vs. Standard } \\
\text { Care }\end{array}$ & $\begin{array}{l}22.6 \pm 11.5 \mathrm{vs} \\
20.0 \pm 10.6 \mathrm{~min}\end{array}$ & $33^{\circ} \mathrm{C}$ in the ICU & $\begin{array}{l}\geq 18 \text { years of age, in cardiac } \\
\text { arrest, had IV access established, } \\
\text { and still in CA result of trauma } \\
\text { (including hanging), suspected } \\
\text { of intracranial bleeding }\end{array}$ & $\begin{array}{l}\text { Pregnant women, to be already } \\
\text { hypothermic }\left(<34.5^{\circ} \mathrm{C}\right) \text {, or } \\
\text { inpatients in a hospital and } \\
\text { documenting limitations in } \\
\text { resuscitation. }\end{array}$ & $\begin{array}{l}\text { Hospital } \\
\text { dis- } \\
\text { charge }\end{array}$ & $\begin{array}{l}\text { Serum } \\
\text { neuron-specific } \\
\text { enolase } \\
\text { concentration at } \\
24 \mathrm{~h}\end{array}$ \\
\hline $\begin{array}{l}\text { Cronberg } \\
\text { et al. } \\
\text { (2015) } \\
{[25]}\end{array}$ & 939 & Unconscious $(\mathrm{GCS}<8)$ & $\begin{array}{l}\text { Ice-cold fluids, ice-packs, } \\
\text { and intravascular or surface } \\
\mathrm{T}^{\circ} \text { management devices at } \\
\text { the discretion of the site. }\end{array}$ & $\begin{array}{l}20 \text { (15-27) vs. } 20 \\
(14-30) \min \end{array}$ & $33^{\circ} \mathrm{C}$ & $\begin{array}{l}\geq 18 \text { years of age, } \text { OHCA of } \\
\text { cardiac cause with ROSC }\end{array}$ & $\begin{array}{l}\text { ROSC }>240 \mathrm{~min}, \mathrm{CA} \text { with asystole } \\
\text { as the initial rhythm, acute } \\
\text { intracranial hemorrhage or stroke, } \\
\text { and } \mathrm{T}^{\circ}<30^{\circ} \mathrm{C}\end{array}$ & 6 months & 6 months \\
\hline $\begin{array}{l}\text { Kirkegaard } \\
\text { et al. } \\
\text { (2017) } \\
{[26]}\end{array}$ & 355 & OHCA of cardiac origin & $\begin{array}{l}\text { Invasive cooling with } \\
\text { intravascular catheter }\end{array}$ & $\begin{array}{l}\text { 48-h group: } 281 \text { (IQR, } \\
\text { 217-360) vs. } 24-\mathrm{h} \\
\text { group: } 320 \text { (IQR } \\
\text { 241-410) } \min (p=0.01)\end{array}$ & $33^{\circ} \mathrm{C}$ & $\begin{array}{l}17-80 \text { years, ROSC }>20 \text { min, } \\
\text { GCS }>8 \text {, shockable and non } \\
\text { shockable rhythms }\end{array}$ & Patients with unwitnessed asystole & 6 months & 6-months (CPC) \\
\hline $\begin{array}{l}\text { Lascarrou } \\
\text { et al. } \\
(2019) \\
{[22]}\end{array}$ & 584 & $\begin{array}{l}\text { Iintrahospitalary: } 27.4 \% \text {; } \\
\text { OHCA: } 72.6 \% \text {; non-cardiac } \\
\text { cause: } 66 \% \text {; circulatory } \\
\text { shock: } 58 \%\end{array}$ & NR & NR & $\begin{array}{l}33^{\circ} \mathrm{C} \pm 0.5^{\circ} \mathrm{C} \\
\text { for } 24 \mathrm{~h}\end{array}$ & $\begin{array}{l}>18 \text { years, resuscitated from } \\
\text { OHCA with a non shockable } \\
\text { rhythm, GCS }>8\end{array}$ & $\begin{array}{l}\text { Low-flow > } 60 \text { min; hemodynamic } \\
\text { instability, CA > 300 min, } \\
\text { moribund condition, cirrhosis, } \\
\text { pregnancy, previous inclusion in } \\
\text { RCT, no health insurance }\end{array}$ & 3 months & 3 months (CPC) \\
\hline $\begin{array}{l}\text { Lilja et al. } \\
\text { (2015) } \\
{[27]}\end{array}$ & 652 & $\begin{array}{l}\text { Unconscious, } \geq 18 \text { years, } \\
\text { OHCA of cardiac origin, } \\
\text { Controls were patients with } \\
\text { STEMI with percutaneous } \\
\text { coronary intervention }\end{array}$ & $\begin{array}{l}4{ }^{\circ} \mathrm{C} \text { intravenous solutions, } \\
\text { ice-packs, and cooling } \\
\text { devices }\end{array}$ & $\begin{array}{l}20(15-30) 21 \\
(13-30) \mathrm{min}\end{array}$ & $33^{\circ} \mathrm{C}$ for $24 \mathrm{~h}$ & $\begin{array}{l}>18 \text { years, OHCA of cardiac } \\
\text { cause, unconsciousness after } \\
\text { ROSC }\end{array}$ & $\begin{array}{l}\text { Bleeding diathesis, acute } \\
\text { intracranial bleeding, stroke, } \\
\text { asystole, therapy limitations and } \\
\text { Do Not Resuscitate order, } \mathrm{CPC}=3 \\
\text { or } 4, \mathrm{~T}^{\circ}<30^{\circ} \mathrm{C}, \mathrm{SBP}<80 \mathrm{mmHg}\end{array}$ & 3 months & 3 months \\
\hline $\begin{array}{l}\text { Look } \\
\text { et al. } \\
\text { (2018) } \\
{[34]}\end{array}$ & 87 & $\begin{array}{l}\text { Controls: } 42.9 \% \text { initial } \\
\text { rhythm, } 33.3 \% \text { PEA, } 9.5 \% \\
\text { VF/VT. TTM: initial } \\
\text { rhythm: } 26.1 \% \text { vs. } 36.4 \% \text { for } \\
\text { asystole, } 34.8 \% \text { vs. } 31.8 \% \\
\text { for PEA, } 13.0 \% \text { vs. } 9.1 \% \text { for } \\
\text { VF/VT }\end{array}$ & $\begin{array}{l}\text { External cooling: four } \\
\text { water-circulating gel-coated } \\
\text { energy transfer pads placed } \\
\text { on the patient's back, } \\
\text { abdomen, and both thighs. } \\
\text { Surface area ranges: } \\
0.60-0.77 \mathrm{~m}^{2} \text { connected to } \\
\text { an automatic thermostat }\end{array}$ & $\begin{array}{l}\text { Median time in } \\
\text { minutes to first ROSC } \\
\text { (Mean, IQR) Control } \\
24.0(13.5,42.0) \text {, } \\
\text { Intervention external } \\
25.0(15.3,39.3) \text {, } \\
\text { internal 26.0 } \\
(12.3,40.3) \text { min }\end{array}$ & $\begin{array}{l}34^{\circ} \mathrm{C} \text {, after which, } \\
\text { they were } \\
\text { maintained at that } \\
\text { temperature for } 24 \mathrm{~h} \\
\text { before rewarming } \\
\text { passively at } 1^{\circ} \mathrm{C} \\
\text { every } 4 \mathrm{~h}\left(0.25^{\circ} \mathrm{C} / \mathrm{h}\right) \\
\text { to } 36.5^{\circ} \mathrm{C}\end{array}$ & $\begin{array}{l}\text { Sustained ROSC after CA for } \\
>30 \text { min, Age: } 18-80 \text { years, } \\
\text { hemodynamically stable, } \\
\text { SBP > } 90 \mathrm{mmHg}\end{array}$ & $\begin{array}{l}\text { Comatose or unresponsive } \\
\text { post-resuscitation, hypotension, } \\
\text { pregnancy, premorbid status, } \\
\text { bedbound and uncommunicative }\end{array}$ & $\begin{array}{l}\text { Hospital } \\
\text { dis- } \\
\text { charge }\end{array}$ & $\begin{array}{l}\text { Hospital } \\
\text { discharge }\end{array}$ \\
\hline $\begin{array}{l}\text { Lopez- } \\
\text { de-Sa } \\
\text { et al. } \\
(2018) \\
{[23]}\end{array}$ & 150 & $\begin{array}{l}\text { AED differed among } \\
\text { groups: } 32^{\circ} \mathrm{C} \text { group: } 13.5 \% \\
\text { vs. } 33^{\circ} \mathrm{C} \text { group: } 34.7 \% \text { vs. } \\
34^{\circ} \mathrm{C} \text { group: } 28.6 \% \text {, } \\
(p=0.03), \text { Invasive coronary } \\
\text { angiography: } 61.5 \% \text { vs. } \\
81.6 \% \text { vs. } 73.5 \%(p=0.08) .\end{array}$ & NR & $\begin{array}{l}32^{\circ} \mathrm{C} 28.9 \pm 15.9,33 \\
{ }^{\circ} \mathrm{C} 26.3 \pm 14.1,34^{\circ} \mathrm{C} \\
25.7 \pm 11.6 \mathrm{~min}\end{array}$ & $\begin{array}{l}32{ }^{\circ} \mathrm{C}, 33^{\circ} \mathrm{C}, \\
34^{\circ} \mathrm{C}\end{array}$ & $\begin{array}{l}\text { Signed consent, } 18-80 \text { years old, } \\
\text { OHCA of cardiac cause, } \\
\text { sustained ROSC, initial } \\
\text { shockable cardiac rhythm, lack } \\
\text { of meaningful response at } \\
\text { arrival to hospital, } \\
\text { non-traumatic CA, } \\
\text { SBP > } 90 \text { Hgmm for } 30 \text { min } \\
\text { post-ROSC }\end{array}$ & $\begin{array}{l}\text { Traumatic/toxicological CA, } \\
\text { pregnancy, Do Not Resuscitate } \\
\text { order, femoral venous access } \\
\text { contraindication, neuromuscular } \\
\text { blocking agents prior to assesment, } \\
\text { incomplete neurological } \\
\text { evaluation, } \mathrm{T}^{\circ}<34^{\circ} \mathrm{C} \text {, current IVC } \\
\text { filter, neurological illness, } \\
\text { functional disabilities, intracranial } \\
\text { bleeding, acute stroke, terminal } \\
\text { illness, other RCT }\end{array}$ & 3 months & 3 months \\
\hline
\end{tabular}


Table 1. Cont

\begin{tabular}{|c|c|c|c|c|c|c|c|c|c|}
\hline $\begin{array}{l}\text { Author } \\
\text { (Year) }\end{array}$ & $\mathbf{N}$ & $\begin{array}{c}\text { Baseline } \\
\text { Characteristics }\end{array}$ & $\begin{array}{l}\text { Cooling } \\
\text { System }\end{array}$ & $\begin{array}{c}\text { Mean Time } \\
\text { Reaching } \\
\text { Target Temperature }\end{array}$ & $\begin{array}{c}\text { Target } \\
\text { Temperature }\end{array}$ & $\begin{array}{l}\text { Inclusion } \\
\text { Criteria }\end{array}$ & $\begin{array}{l}\text { Exclusion } \\
\text { Criteria }\end{array}$ & $\begin{array}{l}\text { Time of } \\
\text { Evaluat- } \\
\text { ing } \\
\text { Survival }\end{array}$ & $\begin{array}{l}\text { Time of } \\
\text { Evaluating } \\
\text { Neurological } \\
\text { Outcome }\end{array}$ \\
\hline $\begin{array}{l}\text { Maynard } \\
\text { et al. } \\
\text { (2015) } \\
{[30]}\end{array}$ & 508 & $\begin{array}{l}37 \% \text { were discharged alive } \\
\text { from the hospital, } 58 \% \text { had } \\
\text { CPC }=1 \text { or } 2,50 \% \text { had MRS: } \\
\text { slight disability or better }\end{array}$ & $\begin{array}{l}\text { Rapid infusion of } 2 \mathrm{~L} \text { of } \\
4^{\circ} \mathrm{C} \text { normal saline, Drug: } \\
\text { Rapid infusion of cold } \\
\text { normal saline }\end{array}$ & NR & $33^{\circ} \mathrm{C}$ vs. $36^{\circ} \mathrm{C}$ & $\begin{array}{l}\text { Successful resuscitation from } \\
\text { OHCA (palpable pulse) }\end{array}$ & Traumatic cause & 12 months & $\begin{array}{l}\text { Hospital } \\
\text { discharge (CPC) }\end{array}$ \\
\hline $\begin{array}{l}\text { Mérei } \\
\text { et al. } \\
\text { (2015) } \\
{[35]}\end{array}$ & 57 & $\begin{array}{l}33 \% \text { female, mean age: } \\
62 \text { years, CPR: } 14.5 \mathrm{~min}, \mathrm{TH}: \\
38 \%\end{array}$ & $\begin{array}{l}2 \mathrm{~L} \text { of intravascular saline at } \\
4{ }^{\circ} \mathrm{C} \text { vs. standard care and } \\
\text { additional external cooling } \\
\text { (cooler blocks over great } \\
\text { blood vessels) }\end{array}$ & NR & $\begin{array}{l}33^{\circ} \mathrm{C} \text { vs. } 36^{\circ} \mathrm{C} \text { for } \\
24 \mathrm{~h}\end{array}$ & $\begin{array}{l}\text { Patients treated on the ICU of } \\
\text { University of Pécs between June } \\
\text { 2009-February } 2012 \text { after CPR, } \\
\text { ROSC }\end{array}$ & NR & NR & 1 month \\
\hline $\begin{array}{l}\text { Nordberg } \\
\text { et al. } \\
\text { (2019) } \\
{[24]}\end{array}$ & 677 & $\begin{array}{l}\text { Median time: } 25 \text { min after } \\
\text { ROSC, median } \mathrm{T}^{\circ}: \\
\text { intervention group: } 34.6^{\circ} \mathrm{C} \\
\text { vs. control group } 35.8^{\circ} \mathrm{C} \\
(p<0.001)\end{array}$ & $\begin{array}{l}\text { Mixture of air or oxygen } \\
\text { and a liquid coolant via } \\
\text { nasal catheters }\end{array}$ & $\begin{array}{l}\text { Intervention group: } \\
105 \text { vs. } 182 \text { min in the } \\
\text { control group } \\
(p<0.001)\end{array}$ & $33 \pm 1^{\circ} \mathrm{C}$ & $\begin{array}{l}\text { Witnessed CA, } \\
18-80 \text { years }\end{array}$ & $\begin{array}{l}\text { Trauma cause, bleeding, drug } \\
\text { overdose, cerebrovascular } \\
\text { accident, drowning, smoke } \\
\text { inhalation, electrocution, hanging, } \\
\text { hypothermic, nasal anatomic } \\
\text { barrier, Do Not Resuscitate order, } \\
\text { terminal disease, pregnancy, } \\
\text { coagulopathy, supplemental } \\
\text { oxygen, EMS }>15 \text { min }\end{array}$ & 3 months & $\begin{array}{l}3 \text { months (CPC } 1 \\
\text { or 2) }\end{array}$ \\
\hline $\begin{array}{l}\text { Pang } \\
\text { et al. } \\
\text { (2016) } \\
{[28]}\end{array}$ & 21 & $\begin{array}{l}\text { Ventricular } \\
\text { tachycardia/ventricular } \\
\text { fibrillation: } 33.3 \% \text {, } \\
\text { pulseless: } 47.6 \% \text {, asystole: } \\
19.0 \% \text {, CPR and ECLS } \\
\text { duration: } 25.7 \text { min and } \\
\text { 4.4 days }\end{array}$ & $\begin{array}{l}\text { ECLS via percutaneous } \\
\text { cannulation of the common } \\
\text { femoral artery. } \\
\text { Extracorporeal centrifugal } \\
\text { pump, oxygenator and heat } \\
\text { exchanger }\end{array}$ & NR & $34^{\circ} \mathrm{C}$ for $24 \mathrm{~h}$ & $\begin{array}{l}\geq 21 \text { years, CA with ECLS, } \\
\text { Ventricular fibrillation, } \\
\text { Downtime }<45 \mathrm{~min} \text {, Comatose } \\
\text { patients, Patients not } \\
\text { responding to verbal after } \\
\text { ROSC, ACLS }<60 \mathrm{~min}, \\
\text { mechanical ventilation }\end{array}$ & $\begin{array}{l}\text { Responding to verbal commands } \\
\text { after ROSC, CPR }>45 \mathrm{~min} \text {, } \\
\text { coagulopathy, pregnancy, } \\
\text { premorbid status, } \\
\text { uncommunicative, } \\
\mathrm{T}^{\circ}<30^{\circ} \mathrm{C}\end{array}$ & 6 months, & $\begin{array}{l}\text { Hospital } \\
\text { discharge (CPC) } \\
\text { of } 1-2\end{array}$ \\
\hline $\begin{array}{l}\text { Scales } \\
\text { et al. } \\
\text { (2017) } \\
{[20]}\end{array}$ & 585 & OHCA patients & $\begin{array}{l}\text { Cold saline and ice packs } \\
\text { applied to neck, axillae, and } \\
\text { both groins and infusion of } \\
\text { up to } 2 \mathrm{~L} \text { of cold saline at } \\
4^{\circ} \mathrm{C}\end{array}$ & $\begin{array}{l}\text { Median (IQR) cooling } \\
\text { group } 5.4 \mathrm{~h}(3.0-8.2) \text { vs. } \\
\text { control } 4.8 \mathrm{~h}(2.8-7.7) \\
(p=0.45)\end{array}$ & $32-34^{\circ} \mathrm{C}$ for $6 \mathrm{~h}$ & $\begin{array}{l}\text { EMS-treated OHCA, } \\
\text { age } \geq 18 \text { years, ROSC of } \geq 5 \text { min, } \\
\text { SBP } \geq 100 \mathrm{mmHg} \text {, unresponsive } \\
\text { to verbal stimuli, endotracheal } \\
\text { intubation }\end{array}$ & $\begin{array}{l}\text { Trauma, burn, or exposure HT, } \\
\text { bleeding, sepsis, coagulopathy, Do } \\
\text { Not Resuscitate order, pregnancy, } \\
\text { or prisoner status }\end{array}$ & $\begin{array}{l}\text { Hospital } \\
\text { discharge }\end{array}$ & $\begin{array}{l}\text { Hospital } \\
\text { discharge }\end{array}$ \\
\hline
\end{tabular}

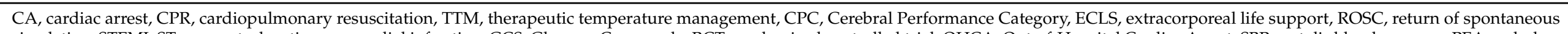

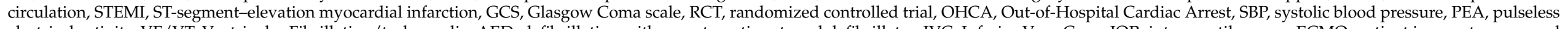

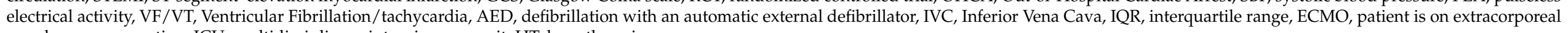
membrane oxygenation, ICU, multidisciplinary intensive care unit, HT, hypothermia. 
Table 2. Characteristic of the selected RCTs in Pediatric patients.

\begin{tabular}{|c|c|c|c|c|c|c|c|c|c|}
\hline $\begin{array}{l}\text { Author } \\
\text { (Year) }\end{array}$ & $\mathbf{N}$ & $\begin{array}{c}\text { Baseline } \\
\text { Characteristics }\end{array}$ & $\begin{array}{l}\text { Cooling } \\
\text { System }\end{array}$ & $\begin{array}{l}\text { Mean Time Reaching } \\
\text { Target Temperature }\end{array}$ & $\begin{array}{c}\text { Target } \\
\text { Temperature }\end{array}$ & $\begin{array}{c}\text { Inclusion } \\
\text { Criteria }\end{array}$ & $\begin{array}{l}\text { Exclusion } \\
\text { Criteria }\end{array}$ & $\begin{array}{l}\text { Time of } \\
\text { Evaluating } \\
\text { Survival }\end{array}$ & $\begin{array}{c}\text { Time of } \\
\text { Evaluating } \\
\text { Neurological } \\
\text { Outcome } \\
\end{array}$ \\
\hline $\begin{array}{l}\text { Fink et al. } \\
\text { (2018) [29] }\end{array}$ & 34 & $\begin{array}{l}88 \% \text { asphyxia, } 82 \% \text { OHCA, } \\
\text { CPR duration: } 20 \text { (11.5, } \\
\text { 30.0) min }\end{array}$ & $\begin{array}{l}\text { Cooling blanket, cold saline } \\
\text { infusion, cold packs, room } \\
\mathrm{T}^{\circ} \text { regulation, and tepid } \\
\text { bath }\end{array}$ & $\begin{array}{l}30(27-33)(30.8 \pm 5.0) \mathrm{h} \\
\text { and } 78(75-79) \\
(76.6 \pm 24.1) \mathrm{h} \text { for the } 24 \\
\text { and } 72 \mathrm{~h} \text { groups, } \\
\text { respectively }\end{array}$ & $33 \pm 1^{\circ} \mathrm{C}$ & $\begin{array}{l}\text { Arterial or venous catheter, } \\
\text { GCS } \leq 8 \text {, HT initiated }\end{array}$ & $\begin{array}{l}\text { Do Not Resuscitate status, } \\
\text { pregnancy, contraindication } \\
\text { for MRI, acute brain disease, } \\
\text { brain death evaluation, } \\
\text { metabolic disease affecting } \\
\text { brain, active hemorrhage, } \\
\text { coagulation defect }\end{array}$ & 6 months & $\begin{array}{l}\text { Neuron-specific } \\
\text { enolase on day } 7 \\
\text { (post-rewarming) }\end{array}$ \\
\hline $\begin{array}{l}\text { Meert et al. } \\
\text { (2016) [21] }\end{array}$ & 54 & $\begin{array}{l}48 \mathrm{~h}-18 \text { years, OHCA with } \\
\mathrm{CPR} \geq 2 \text { min, required } \\
\text { mechanical ventilation after } \\
\text { ROSC }\end{array}$ & $\begin{array}{l}\text { Surface cooling using a } \\
\text { Blanketrol III cooling unit }\end{array}$ & $\begin{array}{l}\text { Median }(\mathrm{Q} 1, \mathrm{Q} 3) \mathrm{HT} \\
\text { Group 5.8 (5.3, 6.3), } \\
\text { Normothermia Group } 6.1 \\
(5.3,7.0)(\mathrm{h})\end{array}$ & $33^{\circ} \mathrm{C}$ for $48 \mathrm{~h}$ & $\begin{array}{l}<1 \text { year at OHCA and ALTE } \\
\text { as the aetiology of arrest }\end{array}$ & $\begin{array}{l}\text { Inability to be randomised } \\
\text { within six hours of return of } \\
\text { circulation, GCS }=5 \text { or } 6.21 \text {, } \\
\text { aggressive treatment, } \\
\text { trauma cause CA. }\end{array}$ & 28 days & 12 months (VABS-II) \\
\hline $\begin{array}{l}\text { Moler et al. } \\
\text { (2015) [31] }\end{array}$ & 295 & $\begin{array}{l}\text { Ventricular fibrillation or } \\
\text { ventricular tachycardia: } 8 \%\end{array}$ & $\begin{array}{l}\text { Pharmacological HT } \\
\text { (paralyzed and sedated), } \\
\text { Blanketrol III temperature } \\
\text { management unit (blankets } \\
\text { applied anteriorly and } \\
\text { posteriorly) }\end{array}$ & $\begin{array}{l}\text { HT group: } 5.9 \text { h (IQR: } \\
5.2 \text {-6.7) vs. } \\
\text { normothermia group: } \\
5.8 \text { h (IQR: } 5.0-6.4 \text { ) }\end{array}$ & $\begin{array}{l}33.0^{\circ} \mathrm{C} \\
(32.0-34.0) \text { for } \\
48 \mathrm{~h}\end{array}$ & $\begin{array}{l}48 \mathrm{~h}, 18 \text { years of age, CA } \\
\text { with CPR }=2 \text { min and } \\
\text { mechanical ventilation after } \\
\text { ROSC }\end{array}$ & $\begin{array}{l}\text { Inability to randomization } \\
\text { within } 6 \text { h after ROSC, GCS } \\
\text { motor-response subscale: } 5 \\
\text { or } 6\end{array}$ & 12 months & 12 months (VABS-II) \\
\hline $\begin{array}{l}\text { Moler et al. } \\
\text { (2017) [32] }\end{array}$ & 329 & $\begin{array}{l}\text { Median age: } 1 \text { year, } 60 \% \\
\text { male, } 91 \% \text { preexisting } \\
\text { medical condition, } 57 \% \\
\text { bradycardia, } 10 \% \\
\text { fibrillation or ventricular } \\
\text { tachycardia, } 93 \% \text { hospital } \\
\text { CA }\end{array}$ & $\begin{array}{l}\text { Blanketrol III } \\
\text { temperature-management } \\
\text { unit applied anteriorly and } \\
\text { posteriorly }\end{array}$ & $\begin{array}{l}\text { CPR median duration } \\
22.0 \text { min (IQR 7.0-47.0) }\end{array}$ & $\begin{array}{l}33.0^{\circ} \mathrm{C} \\
(32.0-34.0) \text { for } \\
48 \mathrm{~h}\end{array}$ & $\begin{array}{l}48 \mathrm{~h}, 18 \text { years of age CA in a } \\
\text { hospital, RCP }>2 \text { min with } \\
\text { mechanical ventilation after } \\
\text { ROSC }\end{array}$ & $\begin{array}{l}\text { GCS motor-response } \\
\text { subscale }=5 \text { or } 6 \text {, inability } \\
\text { to randomization } 6 \mathrm{~h} \text { after } \\
\text { ROSC, bleeding, a } \\
\text { preexisting illness, life } \\
\text { expectancy }<12 \text { months, } \\
\text { aggressive treatment }\end{array}$ & 12 months & 12 months (VABS-II) \\
\hline $\begin{array}{l}\text { Silverstein } \\
\text { et al. (2016) } \\
\text { [33] }\end{array}$ & 295 & $\begin{array}{l}\text { CA etiology: respiratory } \\
(66 \%) \text {, initial EKG rhythm: } \\
\text { asystole }(66 \%), \text { bradycardia } \\
(34 \%), \text { pulseless electrical } \\
\text { activity }(36 \%)\end{array}$ & $\begin{array}{l}\text { Blanketrol III } \\
\text { temperature-management } \\
\text { unit applied anteriorly and } \\
\text { posteriorly }\end{array}$ & NR & $\begin{array}{l}33^{\circ} \mathrm{C} \pm 1^{\circ} \mathrm{C} \text { for } \\
48 \mathrm{~h}\end{array}$ & $\begin{array}{l}\mathrm{CPR}>2 \text { min with ROSC, } 48 \\
\mathrm{~h}, 18 \text { years, continuous } \\
\text { mechanical ventilation } \\
\text { requirement unplanned CA }\end{array}$ & $\begin{array}{l}\text { Speaking barriers, ECMO } \\
\text { when CA } \geq 2 \mathrm{ug} / \mathrm{kg} / \mathrm{min} \\
\text { infusion of epinephrine or } \\
\text { norepinephrine, GCS }=5-6 \text {, } \\
\text { prior CA with CPR }>2 \text { min, } \\
\text { life expectancy }<12 \text { months, } \\
\text { Do Not Resuscitate order }\end{array}$ & 1 year & $\begin{array}{l}12 \text { months (VABS-II } \\
\text { score } \geq 70 \text { ) }\end{array}$ \\
\hline
\end{tabular}

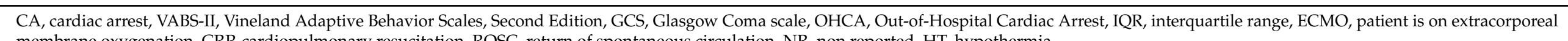
membrane oxygenation, CRP, cardiopulmonary resucitation, ROSC, return of spontaneous circulation, NR, non reported, HT, hypothermia. 
Table 3. Main results of included RCTs in adult population.

\begin{tabular}{|c|c|c|c|c|}
\hline Author (Year) & $\mathbf{N}$ & Aim & Intervention & Results \\
\hline $\begin{array}{l}\text { Bernard et al. (2016) } \\
\text { [19] }\end{array}$ & 1198 & $\begin{array}{l}\text { To measure survival rate at hospital discharge and time to return to } \\
\text { a spontaneous circulation after CA }\end{array}$ & $\begin{array}{l}\text { CPR + TTM vs. CPR + } 2 \mathrm{~L} \text { of cold saline perfusion during an } \\
\text { out-of-hospital CRP }\end{array}$ & $\begin{array}{l}\text { TTM during CPR has no effect on survival rate and may decrease } \\
\text { spontaneous circulation recovery in patients with shockable } \\
\text { rhythms }\end{array}$ \\
\hline $\begin{array}{l}\text { Cronberg et al. (2015) } \\
\text { [25] }\end{array}$ & 939 & $\begin{array}{l}\text { To evaluate the effects of TTM on survival rate, cognitive status, } \\
\text { functionality, and quality of life }\end{array}$ & $\begin{array}{l}\text { TTM at 33-36 }{ }^{\circ} \mathrm{C} \text {. CPC, mRS, MMSE, ALFI, IQCODE, and SF-36 } \\
\text { scales were measured at } 6 \text { months of follow-up in CA survivors }\end{array}$ & Survival rate and quality of life were similar in both groups \\
\hline $\begin{array}{l}\text { Kirkegaard et al. } \\
\text { (2017) } \\
{[26]}\end{array}$ & 355 & To evaluate effectiveness of 48 and $24 \mathrm{~h}$ of TTM & $\begin{array}{l}48 \mathrm{~h} \text { of TTM at } 33^{\circ} \mathrm{C} \text { vs. } 24 \mathrm{~h} \text { of TTM at } 33^{\circ} \mathrm{C}+0.5^{\circ} \mathrm{C} \text { per hour } \\
\text { rewarming }\end{array}$ & $\begin{array}{l}\text { There were no differences in mortality between groups. Patients in } \\
48 \text { h of TTM group showed more adverse events }\end{array}$ \\
\hline $\begin{array}{l}\text { Lascarrou et al. (2019) } \\
\text { [22] }\end{array}$ & 584 & $\begin{array}{l}\text { To measure the effectiveness of TTM in patients in a non-shockable } \\
\text { rhythm }\end{array}$ & $\begin{array}{l}\text { Patients were assigned to } 33^{\circ} \mathrm{C} \text { TTM for } 24 \mathrm{~h} \text { vs. normothermia } \\
\text { treatment at } 37^{\circ} \mathrm{C}\end{array}$ & $\begin{array}{l}\text { TTM group patients showed higher survival and better } \\
\text { neurological status rates than the normothermia group }\end{array}$ \\
\hline $\begin{array}{l}\text { Lilja et al. (2015) } \\
\text { [27] }\end{array}$ & 652 & $\begin{array}{l}\text { To assess 6-month neurological status in patients who received } \\
\text { TTM at } 33-36^{\circ} \mathrm{C} \text { [19] compared to MINOCA patients. }\end{array}$ & $\begin{array}{l}\text { RBMT-3, FAB, Symbols and Digits Test, and HADS scales were } \\
\text { used to compare neurological status between groups. }\end{array}$ & $\begin{array}{l}\text { Cognitive function was comparable in CA survivors and MINOCA } \\
\text { groups }\end{array}$ \\
\hline $\begin{array}{l}\text { Look et al. (2018) } \\
\text { [34] }\end{array}$ & 87 & $\begin{array}{l}\text { To analyze survival rate after hospital discharge and neurological } \\
\text { status in survivors of CA after undergoing intravascular and } \\
\text { surface cooling devices for TTM. }\end{array}$ & $\begin{array}{l}\text { Patients were randomized to intravascular and surface cooling } \\
\text { devices for TTM or to non- therapeutic hypothermia treatment. }\end{array}$ & $\begin{array}{l}\text { Intravascular TTM showed more accurate temperature control, } \\
\text { and non-significant higher rates of survival were found in } \\
\text { comparison with surface cooling. }\end{array}$ \\
\hline $\begin{array}{l}\text { Lopez-de-Sa et al. } \\
\text { (2018) } \\
{[23]}\end{array}$ & 150 & $\begin{array}{l}\text { To compare the effect of different temperatures of TTM on } \\
\text { neurological status in out-of-hospital CA }\end{array}$ & $\begin{array}{l}\text { Comatose patients underwent to TTM of } 32^{\circ} \mathrm{C}, 33^{\circ} \mathrm{C} \text {, and } 34^{\circ} \mathrm{C} \\
\text { for } 24 \mathrm{~h} \text {. }\end{array}$ & $\begin{array}{l}\text { There was no significant impact on neurological status and } \\
\text { survival rate. }\end{array}$ \\
\hline $\begin{array}{l}\text { Maynard et al. (2015) } \\
\text { [30] }\end{array}$ & 508 & $\begin{array}{l}\text { Effect of prehospital induction of mild hypothermia on 3-month } \\
\text { neurological status and 1-year survival among out-of-hospital CA } \\
\text { treated with out-of-hospital TTM. }\end{array}$ & $\begin{array}{l}\text { Cerebral Performance Category (CPC) and Modified Rankin Scale } \\
\text { (mRS) were measured by telephone call to compare neurological } \\
\text { status between patients treated with out-of-hospital TTM ( } 2 \text { L of } \\
\text { intravascular saline at } 4^{\circ} \mathrm{C} \text { after CPR) vs. standard care after } \\
\text { resuscitation from CA. }\end{array}$ & $\begin{array}{l}\text { TTM showed no improvement on the neurological status and } \\
\text { survival rate. }\end{array}$ \\
\hline $\begin{array}{l}\text { Mérei et al. (2015) } \\
\text { [35] }\end{array}$ & 57 & $\begin{array}{l}\text { To evaluate } 30 \text {-day survival, TTM efficacy and serum levels of } \\
\text { S100B protein as a prognostic biomarker. }\end{array}$ & $\begin{array}{l}\text { Blood samples of } 20 \mathrm{CA} \text { patients randomly selected from TTM of } \\
32-34^{\circ} \mathrm{C} \text { and standard care groups were taken at admission and } \\
\text { after } 12 \text { and } 36 \mathrm{~h} \text { after recovery to measure S100B levels. }\end{array}$ & TTM showed no improvement on survival and S100B serum levels. \\
\hline $\begin{array}{l}\text { Nordberg et al. (2019) } \\
\text { [24] }\end{array}$ & 677 & $\begin{array}{l}\text { To determine whether prehospital trans-nasal evaporative } \\
\text { intra-arrest cooling improves survival with good neurologic } \\
\text { outcomes compared with cooling initiated after hospital arrival. }\end{array}$ & $\begin{array}{l}\text { Patients were randomly assigned to receive trans-nasal } \\
\text { evaporative intra-arrest cooling or standard care. Both groups } \\
\text { received TTM at } 32{ }^{\circ} \mathrm{C} \text { to } 34^{\circ} \mathrm{C} \text { for } 24 \mathrm{~h} \text {. }\end{array}$ & $\begin{array}{l}\text { Trans-nasal evaporative intra-arrest cooling did not result in a } \\
\text { statistically significant improvement in survival with good } \\
\text { neurologic outcome, however, those patients reached target } \mathrm{T}^{\circ} \\
\text { earlier. }\end{array}$ \\
\hline $\begin{array}{l}\text { Pang et al. (2016) } \\
\text { [28] }\end{array}$ & 21 & $\begin{array}{l}\text { To evaluate safety and efficacy of TTM in patients undergoing } \\
\text { ECLS after CA. }\end{array}$ & $\begin{array}{l}\text { Comparison of a control group of patients with ECLS treated with } \\
\text { normothermia }\left(37^{\circ} \mathrm{C}\right) \text { vs. TTM at } 34^{\circ} \mathrm{C} \text { for } 24 \mathrm{~h} .\end{array}$ & $\begin{array}{l}\text { TTM can be applied safely in ECLS patients. No significant } \\
\text { differences were found in terms of survival or neurological status. }\end{array}$ \\
\hline $\begin{array}{l}\text { Scales et al. (2017) } \\
\text { [20] }\end{array}$ & 585 & $\begin{array}{l}\text { To evaluate if pre-hospital cooling (target temperature of } 32-34{ }^{\circ} \mathrm{C} \\
\text { within } 6 \mathrm{~h} \text { of hospital arrival) leads to higher rates of successful } \\
\text { TTM. }\end{array}$ & $\begin{array}{l}\text { Patients were randomized to receive prehospital cooling (initiated } \\
5 \text { min after ROSC) or usual resuscitation and transport. Both } \\
\text { groups received TTM in the critical care unit. }\end{array}$ & $\begin{array}{l}\text { Prehospital cooling after ROSC did not increase rates of achieving } \\
\text { a target temperature of } 32-34^{\circ} \mathrm{C} \text { within } 6 \mathrm{~h} \text { of hospital arrival, but } \\
\text { it was safe and increased application of TTM in hospital. }\end{array}$ \\
\hline
\end{tabular}

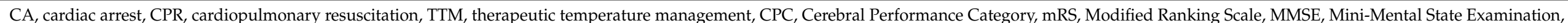

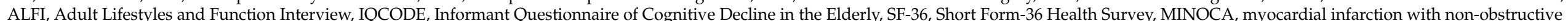

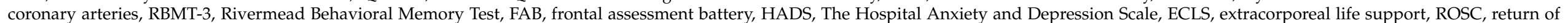
spontaneous circulation. 
Table 4. Main results of the included RCTs in Pediatric patients.

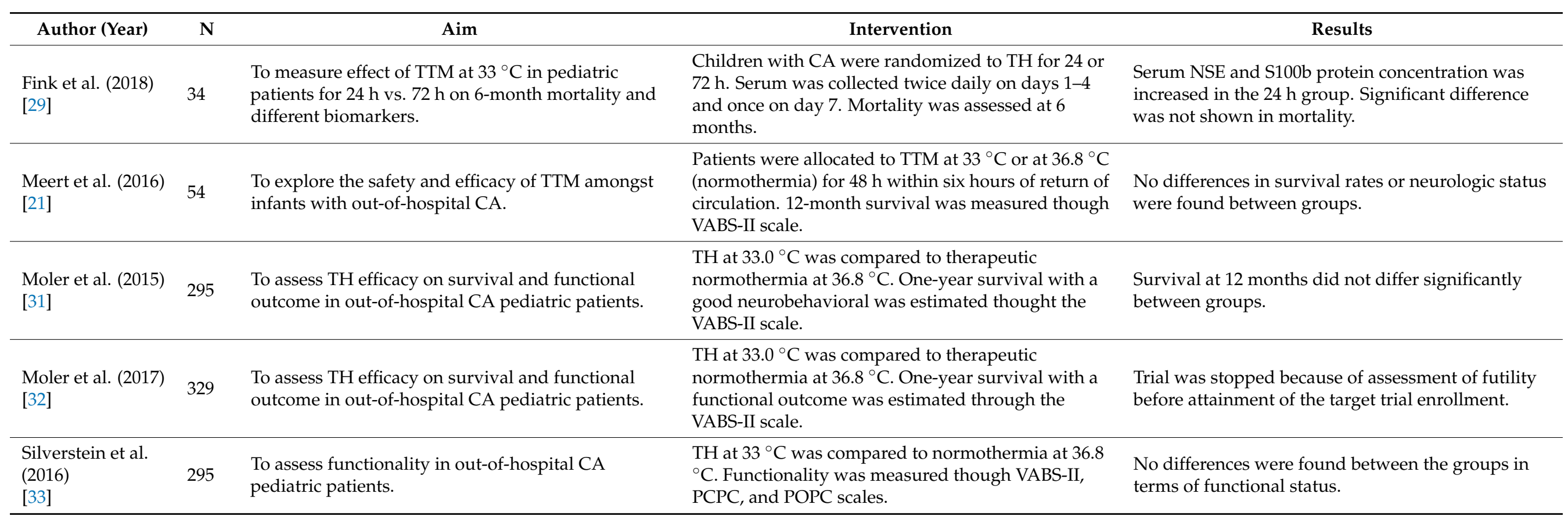

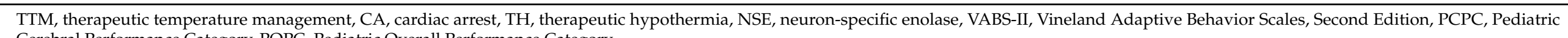
Cerebral Performance Category, POPC, Pediatric Overall Performance Category. 


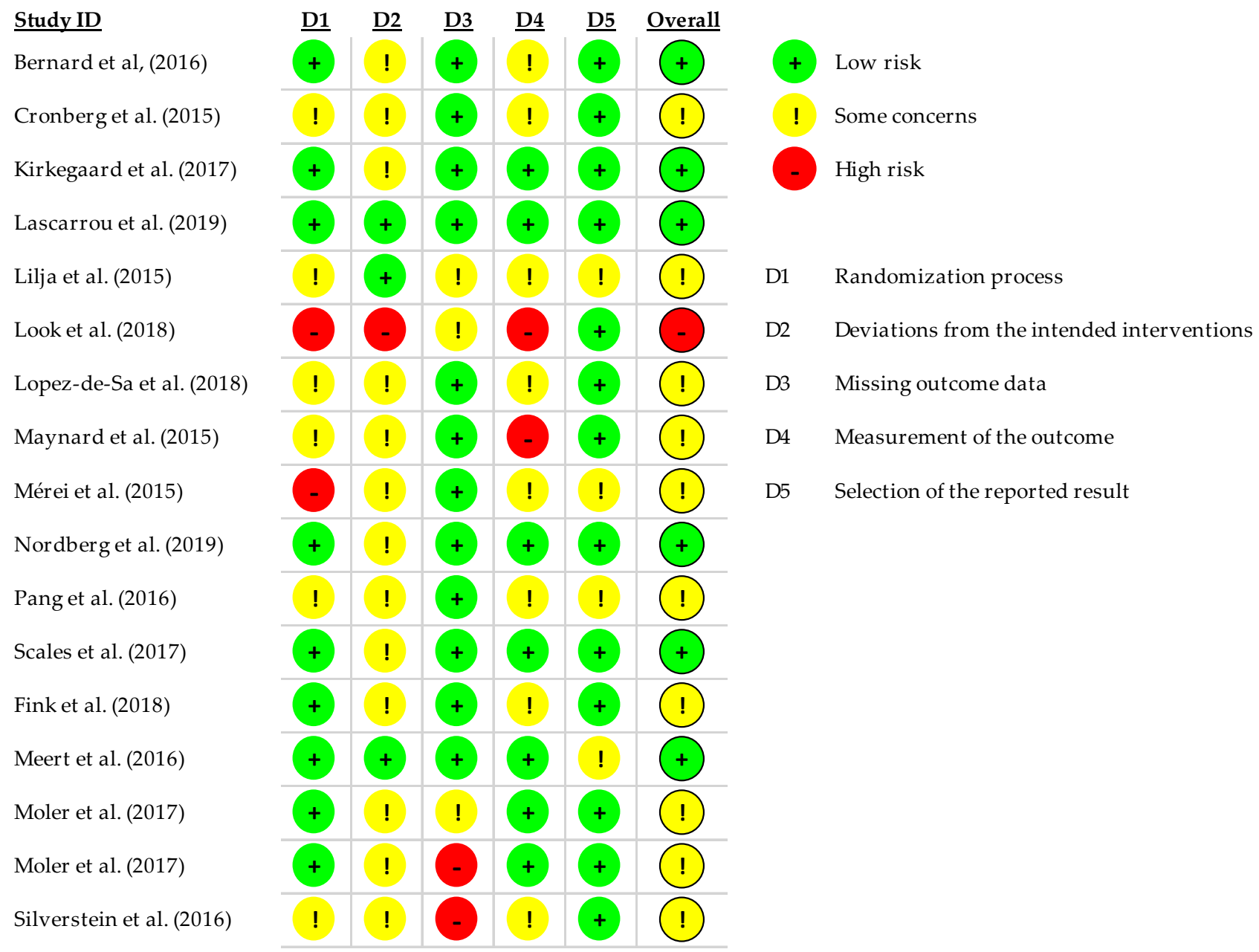

Figure 2. Risk of bias in included trials on effectiveness of therapeutic hypothermia after cardiac arrest on the neurological outcome.

\section{Discussion}

\subsection{Therapeutic Hypothermia in Adults}

Cardiorespiratory arrest data in adults was homogeneous. CA patients were aged between 61 and 68 years. Most studies reported a higher proportion of men (74.8-85.7\%) [19,24-27] Nonetheless, in other studies, that proportion dropped to $65 \%[20,22]$. Two studies highlighted that $60 \%$ of cases of cardiorespiratory arrest are witnessed by someone $[19,20]$. In contrast, in two studies, this percentage rose to $90 \%$ [22,27]. However, only $46 \%$ of CA are attended by witnesses or non-medical personnel before emergency services arrival [20]. Regarding the etiology of CA, $85-96 \%$ present a probable cardiac cause $[18,19]$. However, the study by Lascarrout et al. that analyzed non-shockable rhythms CA reported asphyxia as the main cause of CA in $56 \%$ of cases [22].

A total of $41 \%$ to $47 \%$ of patients showed shockable rhythm when a defibrillator was connected $[19,20,24]$. Cronberg et al. reported that approximately $93-94 \%$ of patients who suffered a CA and survived at least six months had showed an initial shockable rhythm [25]. In addition, Lascarrout's study showed that only $17.7 \%$ of patients who suffered a CA with an initial non-shockable rhythm were alive at 90 days [22]. Furthermore, non-shockable rhythms are associated with worse neurologic status [36]. Results from another study stated that those patients that were defibrillated by a witness of the CA increased by $67 \%$ the chance of ending with a good neurological state at discharge [23]. In this context, 
early defibrillation is essential since it is the most effective treatment against ventricular fibrillation and increases survival rates of CA patients [37]. A rang of $54.4-57 \%$ of the reported CA cases occurred at home and $38.7 \%$ in a public place $[26,27]$. Studies indicated that angiography procedure appears to be the most frequent post-CA intervention (56-82\% of cases). TTM can be applied simultaneously with angiography, so the application of one technique should not be delayed by the other [26,27].

\subsection{Targeted Temperature Management}

Results from the study by Lascarrout et al. reported better neurologic status 90 days after resuscitation in $\mathrm{CA}$ patients treated with $\mathrm{TH}$ at $33^{\circ} \mathrm{C}$ compared to the control group using the CPC Scale [22]. In accordance with this evidence, patients with initial nonshockable rhythms may also benefit from TH. However, in this study, patients in the control group were not excluded if they experienced fever (temperature $>38^{\circ} \mathrm{C}$ ). This fact could be responsible for harmful effects, thereby overestimating the beneficial effects of TTM. Hyperthermia prevention could have been enough to achieve these benefits.

In contrast, Lilja et al. compared the effectiveness of $\mathrm{TH}$ at $33^{\circ} \mathrm{C}$ and $36{ }^{\circ} \mathrm{C}$, concluding that no benefits on neurologic status were found according to the CPC scale [27]. Moreover, these results were in accordance with another study that compared $\mathrm{TH}$ at $32{ }^{\circ} \mathrm{C}, 33{ }^{\circ} \mathrm{C}$, and $34{ }^{\circ} \mathrm{C}$, and no differences in neurologic status were found [27]. It is worth mentioning that the sample size in that study was a great deal smaller, whereby results should be analyzed with caution.

Cronberg et al. concluded that there were no significant differences in any of the scales that measured cognitive function, quality of life, and neurological status in patients who had suffered a CA and had been treated with $\mathrm{TH}$ at $33^{\circ} \mathrm{C}$ or $36^{\circ} \mathrm{C}$ [25].

Therefore, the evidence appears to indicate that the prevention of hyperthermia and TTM at $36{ }^{\circ} \mathrm{C}$ are more effective than TH application. Nonetheless, it is possible that specific subgroups of patients that have suffered a CA may benefit from $\mathrm{TH}$.

\subsubsection{Adverse Events}

As the Lopez-de-Sa et al. study indicated, the group assigned to $\mathrm{TH}$ at $32{ }^{\circ} \mathrm{C}$ showed a lower risk of respiratory infections, however, they suffered arrhythmias more frequently, specifically bradycardias [35]. Results from another RCT came to similar conclusions when patients treated with TH at 32 and $34{ }^{\circ} \mathrm{C}$ were compared. Patients assigned to the $32{ }^{\circ} \mathrm{C}$ TH group showed lower prevalence of seizures but more cases of bradycardia [38]. Nevertheless, one study concluded that bradycardia during TH intervention was associated with better outcomes at hospital discharge [39].

On the other hand, another frequent adverse effect associated with TH reported by Mérei et al. is the likelihood of developing thrombocytosis [35].

These adverse events are in line with the current evidence [16]. Additionally, it has been shown that these events have no serious health consequences, so TH is considered a safe therapy, and it can be implemented together with other interventions, such as extracorporeal life support (ECLS), as Pang et al. reported [28].

Bernard et al. concluded that patients that received TH during their CA showed higher rates of pulmonary edema (10\%) than those who received standard treatment (4.5\%) [19]. Thus, ice-cold saline for the induction of mild hypothermia is discouraged during CA. Another study pointed that saline infusion leads to a rise central venous pressure (CVP), compromising coronary perfusion [40].

On the contrary, Scales et al. reported no adverse effects associated to TH application five minutes after CA. Furthermore, pulmonary edema was lower in the group that received out-of-hospital TH [20]. One explanation could be that only $72 \%$ received intravenous outof-hospital TH and that the volume infused was smaller than in the Bernard et al. study.

None of the studies included in this review showed significant differences in survival rate. 


\subsubsection{Prognostic Factors}

Mérei et al. observed higher rates of mortality in patients with lower Glasgow Coma scale (GCS) score at the time of admission to intensive care [35]. They concluded that neurologic status at discharge was a long-term indicator of neurologic status and survival [37]. This could mean that the chances of improving neurological status of CA patients is very low.

\subsubsection{Quality of Life in CA Patients}

Cronberg et al. reported in their study that $49.4 \%$ of CA survivors were active workers before the event, and only 30\% continued working after the event [25]. Therefore, it can be concluded that surviving a CA will likely affect the daily life of these patients due to CA sequelae.

\subsection{Targeted Hypothermia in Out-of-Hospital CA Patients}

The devices most frequently used in the studies analyzing TH effectiveness in the out-of-hospital environment are cold saline infusion and intranasal evaporative catheter.

Bernard et al. reported that patients who received cold saline infusion during CA took more time to return to spontaneous circulation than those who underwent standardized treatment. In addition, mortality rates were higher in CA patients treated with TH during CA compared to those who received normal treatment. However, no significant survival differences were found between groups [19]. One possibility is that saline infusion TH treatment could be delaying other procedures aimed at CA reversion. These findings are in line with the study by Maynard et al., where they concluded that patients that received out-of-hospital TH showed similar outcomes in terms of neurological status to patients that were treated with TH after hospital arrival [30]. Moreover, patients who received out-of-hospital TH were more likely to also receive hospital TH according to Scales et al. Nonetheless, this fact did not lead to higher survival rates or better neurologic status [20]. In contrast, the study carried out by Garrett et al. showed that $36.5 \%$ of the CA patients that received TH recovered spontaneous circulation compared to $26.9 \%$ of those who did not receive it. This, however, did not lead to a rise in survival rate [41].

Nordberg et al. concluded that using an intranasal device during CA could reduce body temperature to the target temperature faster than standard care. However, survival rate and neurological outcomes were similar between groups in this case, too [24].

Considering the results of these studies, it seems more reasonable to initiate $\mathrm{TH}$ using intranasal devices rather than cold saline infusion. Moreover, these findings also indicate that TH may possibly not have the beneficial effect on patients that Holzer et al. and Bernard et al. reported in their studies back in $2002[42,43]$.

\subsubsection{Maintenance Phase}

Kirkegaard et al. reported no differences in survival rates between patients treated with TH during 24 or $48 \mathrm{~h}$. However, patients that showed optimal neurologic status at six months after suffering a CA was $5 \%$ higher in the $24-\mathrm{h}$ TH group. Although no significant differences were found, it is not possible to completely dismiss possible beneficial effects. In addition, that study showed higher prevalence of adverse events such as hypotension in the 48-h TH group [26]. Another observational study concluded that increasing TH time led to higher rates of hemorrhage, pneumonia, and arrhythmia [44]. Therefore, a 24-h TH intervention seems more effective.

\subsubsection{Internal and External Cooling Devices}

The study by Look et al. showed that patients treated with internal devices reached higher survival rates than patients that did not receive TH. They also found that those devices allowed for a more accurate temperature control. Nonetheless, survival rates were not statistically different and the insufficient sample size ought to be taken into consideration [34]. 
Another observational study came to a similar conclusions, reporting no significant differences in survival rates in terms of neurological status between patients that received invasive TH in comparison to surface cooling with blankets or ice [45]. Another study also pointed out that invasive device use increases the risk of infection and bleeding [46]. Therefore, internal device use entails a higher risk of adverse events despite offering a more accurate temperature control.

\subsubsection{Risk of Bias}

In order to carry RCTs on TTM, a large enough sample size is required since TH beneficial effect rates may not be higher that $5-10 \%$. In this context, this kind of RCTs are usually multicenter, but in some cases, the choice of certain TH parameters are left to the individual centers, which can lead to important differences between hospitals. In the cases of Kirkegaard et al. and Lascarrout et al., different cooling devices were used in the TH intervention, which could lead to biased results [22,26]. Moreover, studies like those by Mérei et al. or Scales et al. have an insufficient sample size [20,35]. On the other hand, it is not possible to interview all CA survival patients to assess neurologic status at discharge. In the study by Maynard et al., for example, the proportion of patients interviewed was only $73 \%$ [37]. Bernart et al. had to end the study when targeted temperature protocol at the hospitals participating in the trial changed from $33^{\circ} \mathrm{C}$ to $36^{\circ} \mathrm{C}$. That change was imposed after TTM study publication [47]. Subsequently, lower rates of compliance of TH at $33-36{ }^{\circ} \mathrm{C}$ were reported, as were higher rates of fever and worse clinical prognosis. We assessed the risk of bias using the Cochrane risk of bias assessment tool (Higgins et al., 2011), which demonstrated moderate risk of bias in the majority of included studies [18].

As a result, Bray et al. recommended that hospitals that decide to practice $\mathrm{TH}$ at $36^{\circ} \mathrm{C}$ should adequately sedate patients and consider the use of muscle relaxants in order to prevent fever onset [48].

The impossibility of blinding the TH interventions is another determinant of bias.

Finally, the most commonly used scales to measure neurologic status in this kind of patients are CPC and mRS. Rittenberger et al. concluded that those instruments have not been validated, and more precise tools are needed to assess CA survivors [49].

\subsection{Hypothermia in Pediatric Patients}

Mean age of pediatric patients that suffered CA was between 1 and 3.2 years old [30-33]. Most of the studies showed a higher proportion of males (59-66\%) [21,31,32]. However, the study by Fink et al. included $41 \%$ of males [29]. The main cause of CA was respiratory $(72-88 \%)$, with this etiology being more frequent in the pediatric population than in adults. A cardiac etiology was observed in $10 \%$ of cases [29,32]. From the studied CA events, $11-38 \%$ were witnessed, and in $65 \%$ of cases, resuscitation was initiated by non-medical professionals [21,32]. Non-shockable initial rhythms were the most frequent $(72-88 \%$ of cases). Shockable rhythms constituted between 3-7\%, and in 6-15\%, bradycardia was detected $[29,31,32]$. The study by Moler et al. (2017), which analyzed intra-hospital CA, found that $57 \%$ of patients presented bradycardia, $21 \%$ pulseless electrical activity (PEA), $8 \%$ asystole, and $10 \%$ ventricular fibrillation ( $\mathrm{V}-\mathrm{fib}$ ) [32]. One hypothesis is that intrahospital CA are treated immediately, whereas in out-of-hospital Cas, the connection to a defibrillator is somewhat delayed. It should be noted that, according to the results of Moler et al., the main cause of mortality after recovery of spontaneous circulation in out-ofhospital CA is brain death or withdrawal of life support due to poor neurological prognosis in $81 \%$ of cases. That percentage, however, drops to $36 \%$ in the intra-hospital environment. Furthermore, survival rate with good neurological status was $16 \%$ in out-of-hospital CA patients and $38 \%$ hospital patients. This demonstrates how the prognosis of a CA is intimately related to the time between onset of the arrest and initiation of resuscitation measures [31,32]. 
According to the study carried out by Pittl et al., factors associated with survival rate in pediatric patients were cardiac etiology, ventricular fibrillation initial rhythm, shorter resuscitation, lower epinephrine dose, and weekday and daytime events [46].

\subsubsection{Targeted Temperature Management (TTM)}

In the studies by Moler et al. comparing out-of-hospital and intra-hospital TTM at 33 and $36.8^{\circ} \mathrm{C}$ in CA patients, no differences were found in one-year survival rates nor neurological status $[31,32]$. Meert et al. came to the same conclusion when TTM at $33{ }^{\circ} \mathrm{C}$ for $48 \mathrm{~h}$ was applied to the intervention group in comparison to the control group, which received normothermia [21].

\subsubsection{Adverse Events}

Meert et al. reported a lower proportion of patients that required transfusion of blood products among those treated with TTM although they suffered a higher prevalence of hypoglycemias [21]. Nonetheless, these results are probably unreliable, as the sample size was not statistically valid. Furthermore, other studies with an adequate sample size showed no differences in terms of adverse events or greater use of blood products [31,32].

\subsubsection{Maintenance Phase Duration}

The results of the study by Fink et al., which compared TTM for 24 and $72 \mathrm{~h}$, showed a decrease of brain damage biomarkers in the 72-h treatment group. Nevertheless, no differences in survival rates and levels of brain damage were found after six months of follow-up [29]. These findings, notwithstanding, should be taken with care since there were already differences in these biomarkers before the TTM for $24 \mathrm{~h}$ intervention, so its variability cannot be attributed to the treatment. In addition, this study has sampling limitations, too, so its conclusions cannot be extrapolated.

\subsubsection{Risk of Bias}

It has been observed that studies in pediatric populations have much smaller sample sizes than studies in adults, which makes it complicated to generalize their conclusions and increases the risk of bias. This fact also limits the ability to detect the beneficial effects of TTM if rates are low (Figure 2).

This review has certain limitations. On the one hand, the lack of RCTs with a sufficient sample size makes it difficult to reach reliable conclusions on the efficacy of TRM. In addition, it is a systematic review of RCTs published between 2016 and 2020, which may limit the results presented. Nevertheless, it has been observed through the results from previous years in the different databases that the largest number of publications occurred in the inclusion period, except for 2015.

\section{Conclusions}

The evidence on TTM suggest that it is a safe procedure with few and manageable adverse effects in the hospital environment. Furthermore, it can be used in conjunction with post-resuscitation procedures, such as angiography and extracorporeal life support. Regarding TTM use in adult population after CA, no differences were found in comparison with normothermia. However, it is possible that TTM could have beneficial effects on certain subgroups of patients. Studies in pediatric patients did not report significant differences between TTM and normothermia effectiveness even if applied for more than $24 \mathrm{~h}$. However, some studies point that TTM could have a beneficial effect on survival rates and neurological status in both adults and children. Evidence shows that neuroprotection can likely be achieved solely by hyperthermia prevention although more RCTs and metaanalysis of RCT with greater sample sizes are needed to determine if differences exist. Regarding out-of- hospital TTM, the results are controversial since pulmonary edema is associated with intravenous cold saline infusion for hypothermia induction. Intranasal device use appears to be a safer method. Moreover, any type of out-of-hospital TTM 
improves the chances of hospital TTM intervention at admission. However, its effect on survival and neurological status has not been demonstrated. Finally, more accurate scales to assess neurologic status are needed in order to reduce variability and provide more precise results.

Author Contributions: Conceptualization: J.C.S.G. and C.C.G.; methodology: J.C.S.G., B.R.G., R.R.B. and J.C.M.; validation J.C.M. and M.M.T.; formal analysis: J.C.S.G., C.C.G. and J.C.M.; investigation: J.C.S.G. and C.C.G.; original draft preparation B.R.G., J.C.M. and R.R.B.; review and editing: J.C.S.G., J.C.M. and M.M.T.; visualization: J.C.S.G., C.C.G. and B.R.G.; supervision: J.C.S.G., C.C.G. and R.R.B. All authors have read and agreed to the published version of the manuscript.

Funding: This research received no external funding.

Institutional Review Board Statement: Not applicable.

Informed Consent Statement: Not applicable.

Data Availability Statement: Not applicable.

Conflicts of Interest: The authors declare no conflict of interest.

\section{References}

1. Jacobs, I.; Nadkarni, V.; Bahr, J.; Berg, R.A.; Billi, J.E.; Bossaert, L.; Cassan, P.; Coovadia, A.; D’Este, K.; Finn, J.; et al. Cardiac arrest and cardiopulmonary resuscitation outcome reports: Update and simplification of the Utstein templates for resuscitation registries. A statement for healthcare professionals from a task force of the International Liaison Committee on Resuscitation (American Heart Association, European Resuscitation Council, Australian Resuscitation Council, New Zealand Resuscitation Council. Circulation 2004, 110, 3385-3397. [PubMed]

2. Berdowskki, J.; Berg, R.A.; Tijssen, J.G.P.; Koster, R.W. Global Incidences of out-of-Hospital Cardiac Arrest and Survival Rates: Systematic Review of 67 Prospective Studies I Elsevier Enhanced Reader. Available online: https://reader.elsevier.com/reader/ sd/pii/S0300957210004326?token=D23166ACD16312FBB6319D95402CC4CF28F3350CE5A3CC5791E8B26FEC2E2A58829847F3 BAC5070BF5B802BC0223F146 (accessed on 5 September 2020).

3. Porzer, M.; Mrazkova, E.; Homza, M.; Janout, V. Out-of-hospital cardiac arrest. Biomed. Pap. 2017, 161, 348-353. [CrossRef] [PubMed]

4. Luc, G.; Baert, V.; Escutnaire, J.; Genin, M.; Vilhelm, C.; Di Pompéo, C.; El Khoury, C.; Segal, N.; Wiel, E.; Adnet, F.; et al. Epidemiology of out-of-hospital cardiac arrest: A French national incidence and mid-term survival rate study. Anaesth. Crit. Care Pain Med. 2019, 38, 131-135. [CrossRef] [PubMed]

5. Nolan, J.P.; Neumar, R.W.; Adrie, C.; Aibiki, M.; Berg, R.A.; Böttiger, B.W.; Callaway, C.; Clark, R.S.B.; Geocadin, R.G.; Jauch, E.C.; et al. Post-cardiac arrest syndrome: Epidemiology, pathophysiology, treatment, and prognostication. A Scientific Statement from the International Liaison Committee on Resuscitation; the American Heart Association Emergency Cardiovascular Care Committee; the Council on Cardiovascular Surgery and Anesthesia the Council on Cardiopulmonary, Perioperative, and Critical Care the Council on Clinical Cardiology. Resuscitation 2008, 79, 350-379. [CrossRef] [PubMed]

6. Girotra, S.; Chan, P.S.; Bradley, S.M. Post-resuscitation care following out-of-hospital and in-hospital cardiac arrest. Heart 2015, 101, 1943-1949. [CrossRef]

7. Callaway, C.W.; Soar, J.; Aibiki, M.; Böttiger, B.W.; Brooks, S.C.; Deakin, C.D.; Donnino, M.W.; Drajer, S.; Kloeck, W.; Morley, P.T.; et al. Part 4: Advanced life support: 2015 International consensus on cardiopulmonary resuscitation and emergency cardiovascular care science with treatment recommendations. Circulation 2015, 132, S84-S145. [CrossRef] [PubMed]

8. Laver, S.; Farrow, C.; Turner, D.; Nolan, J. Mode of death after admission to an intensive care unit following cardiac arrest. Intensive Care Med. 2004, 30, 2126-2128. [CrossRef]

9. Jennett, B.; Bond, M. Assessment of outcome after severe brain damage. A practical scale. Lancet 1975, 305, 480-484. [CrossRef]

10. Holzer, M. Targeted temperature management for comatose survivors of cardiac arrest. N. Engl. J. Med. 2010, 363, 1256-1264. [CrossRef]

11. Madl, C.; Holzer, M. Brain function after resuscitation from cardiac arrest. Curr. Opin. Crit. Care 2004, 10, 213-217. [CrossRef]

12. Lázaro Paradinas, L. Conocimiento enfermero sobre hipotermia inducida tras parada cardiorrespiratoria: Revisión bibliográfica. Enferm. Intensiva 2012, 23, 17-31. [CrossRef] [PubMed]

13. McCullough, J.N.; Zhang, N.; Reich, D.L.; Juvonen, T.S.; Klein, J.J.; Spielvogel, D.; Ergin, M.A.; Griepp, R.B. Cerebral metabolic suppression during hypothermic circulatory arrest in humans. Ann. Thorac. Surg. 1999, 67, 1895-1899. [CrossRef]

14. Webster, C.M.; Kelly, S.; Koike, M.A.; Chock, V.Y.; Giffard, R.G.; Yenari, M.A. Inflammation and NFkB activation is decreased by hypothermia following global cerebral ischemia. Neurobiol. Dis. 2009, 33, 301-312. [CrossRef] [PubMed]

15. Alzaga, A.G.; Cerdan, M.; Varon, J. Therapeutic hypothermia. Resuscitation 2006, 70, 369-380. [CrossRef] [PubMed]

16. Seder, D.B.; Jarrah, S. Therapeutic Hypothermia for Cardiac Arrest: A Practical Approach. Curr. Neurol. Neurosci. Rep. 2008, 8, 508-517. [CrossRef] 
17. Page, M.J.; McKenzie, J.E.; Bossuyt, P.M.; Boutron, I.; Hoffmann, T.C.; Mulrow, C.D.; Shamseer, L.; Tetzlaff, J.M.; Akl, E.A.; Brennan, S.E.; et al. The PRISMA 2020 statement: An updated guideline for reporting systematic reviews. BMJ 2021, $372, \mathrm{n} 71$. [CrossRef]

18. Higgins, J.P.T.; Altman, D.G.; Gotzsche, P.C.; Juni, P.; Moher, D.; Oxman, A.D.; Savović, J.; Schulz, K.F.; Weeks, L.; Sterne, J.A. The Cochrane Collaboration's tool for assessing risk of bias in randomised trials. BMJ 2011, 343, d5928. [CrossRef]

19. Bernard, S.A.; Smith, K.; Finn, J.; Hein, C.; Grantham, H.; Bray, J.E.; Deasy, C.; Stephenson, M.; Williams, T.A.; Straney, L.D.; et al. Induction of Therapeutic Hypothermia during Out-of-Hospital Cardiac Arrest Using a Rapid Infusion of Cold Saline: The RINSE Trial (Rapid Infusion of Cold Normal Saline). Circulation 2016, 134, 797-805. [CrossRef]

20. Scales, D.C.; Cheskes, S.; Verbeek, P.R.; Pinto, R.; Austin, D.; Brooks, S.C.; Dainty, K.N.; Goncharenko, K.; Mamdani, M.; Thorpe, K.E.; et al. Prehospital cooling to improve successful targeted temperature management after cardiac arrest: A randomized controlled trial. Resuscitation 2017, 121, 187-194. [CrossRef]

21. Meert, K.; Telford, R.; Holubkov, R.; Slomine, B.S.; Christensen, J.R.; Dean, J.M.; Moler, F.W. Exploring the safety and efficacy of targeted temperature management amongst infants with out-of-hospital cardiac arrest due to apparent life threatening events. Resuscitation 2016, 109, 40-48. [CrossRef]

22. Lascarrou, J.B.; Merdji, H.; Le Gouge, A.; Colin, G.; Grillet, G.; Girardie, P.; Coupez, E.; Dequin, P.F.; Cariou, A.; Boulain, T.; et al. Targeted temperature management for cardiac arrest with nonshockable rhythm. N. Engl. J. Med. 2019, 381, 2327-2337. [CrossRef]

23. Lopez-de-Sa, E.; Juarez, M.; Armada, E.; Sanchez-Salado, J.C.; Sanchez, P.L.; Loma-Osorio, P.; Sionis, A.; Monedero, M.C.; Martinez-Sellés, M.; Martín-Benitez, J.C.; et al. A multicentre randomized pilot trial on the effectiveness of different levels of cooling in comatose survivors of out-of-hospital cardiac arrest: The FROST-I trial. Intensive Care Med. 2018, 44, 1807-1815. [CrossRef] [PubMed]

24. Nordberg, P.; Taccone, F.S.; Truhlar, A.; Forsberg, S.; Hollenberg, J.; Jonsson, M.; Cuny, J.; Goldstein, P.; Vermeersch, N.; Higuet, A.; et al. Effect of Trans-Nasal Evaporative Intra-arrest Cooling on Functional Neurologic Outcome in Out-of-Hospital Cardiac Arrest: The PRINCESS Randomized Clinical Trial. JAMA 2019, 321, 1677-1685. [CrossRef]

25. Cronberg, T.; Lilja, G.; Horn, J.; Kjaergaard, J.; Wise, M.P.; Pellis, T.; Hovdenes, J.; Gasche, Y.; Åneman, A.; Stammet, P.; et al. Neurologic Function and Health-Related Quality of Life in Patients Following Targeted Temperature Management at $33{ }^{\circ} \mathrm{C}$ vs. $36{ }^{\circ} \mathrm{C}$ After Out-of-Hospital Cardiac Arrest. JAMA Neurol. 2015, 72, 634-641. [CrossRef]

26. Kirkegaard, H.; Søreide, E.; De Haas, I.; Pettilä, V.; Taccone, F.S.; Arus, U.; Storm, C.; Hassager, C.; Nielsen, J.F.; Sørensen, C.A.; et al. Targeted temperature management for 48 vs. 24 hours and neurologic outcome after out-of-hospital cardiac arrest: A randomized clinical trial. JAMA-J. Am. Med. Assoc. 2017, 318, 341-350. [CrossRef] [PubMed]

27. Lilja, G.; Nielsen, N.; Friberg, H.; Horn, J.; Kjaergaard, J.; Nilsson, F.; Pellis, T.; Wetterslev, J.; Wise, M.P.; Bosch, F.; et al. Cognitive Function in survivors of out-of-hospital cardiac arrest after target temperature management at $33^{\circ} \mathrm{C}$ Versus $36{ }^{\circ} \mathrm{C}$. Circulation 2015, 131, 1340-1349. [CrossRef] [PubMed]

28. Pang, P.Y.K.; Wee, G.H.L.; Hoo, A.E.E.; Sheriff, I.M.T.; Lim, S.L.; Tan, T.E.; Loh, Y.J.; Kerk, K.L.; Sin, Y.K.; Lim, C.H. Therapeutic hypothermia in adult patients receiving extracorporeal life support: Early results of a randomized controlled study. J. Cardiothorac. Surg. 2016, 11, 43. [CrossRef]

29. Fink, E.L.; Clark, R.S.B.; Berger, R.P.; Fabio, A.; Angus, D.C.; Watson, R.S.; Gianakas, J.J.; Panigrahy, A.; Callaway, C.W.; Bell, M.J.; et al. 24 vs. 72 hours of hypothermia for pediatric cardiac arrest: A pilot, randomized controlled trial. Resuscitation 2018, 126, 14-20. [CrossRef]

30. Maynard, C.; Longstreth, W.T.; Nichol, G.; Hallstrom, A.; Kudenchuk, P.J.; Rea, T.; Copass, M.K.; Carlbom, D.; Deem, S.; Olsufka, M.; et al. Effect of prehospital induction of mild hypothermia on 3-month neurological status and 1-year survival among adults with cardiac arrest: Long-term follow-up of a randomized, clinical trial. J. Am. Heart Assoc. 2015, 4, e001693. [CrossRef]

31. Moler, F.W.; Silverstein, F.S.; Holubkov, R.; Slomine, B.S.; Christensen, J.R.; Nadkarni, V.M.; Meert, K.L.; Clark, A.E.; Browning, B.; Pemberton, V.L.; et al. Therapeutic hypothermia after out-of-hospital cardiac arrest in children. N. Engl. J. Med. 2015, 372, 1898-1908. [CrossRef]

32. Moler, F.W.; Silverstein, F.S.; Holubkov, R.; Slomine, B.S.; Christensen, J.R.; Nadkarni, V.M.; Meert, K.L.; Clark, A.E.; Browning, B.; Pemberton, V.L.; et al. Therapeutic hypothermia after in-hospital cardiac arrest in children. Supplementary appendix. N. Engl. J. Med. 2017, 376, 318-329. [CrossRef] [PubMed]

33. Silverstein, F.S.; Slomine, B.S.; Christensen, J.; Holubkov, R.; Page, K.; Dean, J.M.; Moler, F.W. Functional Outcome Trajectories after Out-of-Hospital Pediatric Cardiac Arrest. Crit. Care Med. 2016, 44, e1165-e1174. [CrossRef] [PubMed]

34. Look, X.; Li, H.; Ng, M.; Lim, E.T.S.; Pothiawala, S.; Tan, K.B.K.; Sewa, D.W.; Shahidah, N.; Pek, P.P.; Ong, M.E.H. Randomized controlled trial of internal and external targeted temperature management methods in post- cardiac arrest patients. Am. J. Emerg. Med. 2018, 36, 66-72. [CrossRef] [PubMed]

35. Mérei, Á.; Nagy, B.J.; Woth, G.L.; Zsidó, N.; Lantos, J.; Mühl, D. Effects of therapeutic hypothermia and kinetics of serum protein S100B after cardiopulmonary resuscitation. Signa Vitae-A J. Intensive Care Emerg. Med. 2015, 10, 109-130. [CrossRef]

36. Meaney, P.A.; Nadkarni, V.M.; Kern, K.B.; Indik, J.H.; Halperin, H.R.; Berg, R.A. Rhythms and outcomes of adult in-hospital cardiac arrest. Crit. Care Med. 2010, 38, 101-108. [CrossRef] [PubMed]

37. Eisenberg, M.S.; Hallstrom, A.P.; Copass, M.K.; Bergner, L.; Short, F.; Pierce, J. Treatment of Ventricular Fibrillation: Emergency Medical Technician Defibrillation and Paramedic Services. JAMA J. Am. Med. Assoc. 1984, 251, 1723-1726. [CrossRef] 
38. Lopez-De-Sa, E.; Rey, J.R.; Armada, E.; Salinas, P.; Viana-Tejedor, A.; Espinosa-Garcia, S.; Martinez-Moreno, M.; Corral, E.; Lopez-Sendon, J. Hypothermia in comatose survivors from out-of-hospital cardiac arrest: Pilot trial comparing 2 levels of target temperature. Circulation 2012, 126, 2826-2833. [CrossRef]

39. Stær-Jensen, H.; Sunde, K.; Olasveengen, T.M.; Jacobsen, D.; Drægni, T.; Nakstad, E.R.; Eritsland, J.; Andersen, G.Ø. Bradycardia during therapeutic hypothermia is associated with good neurologic outcome in comatose survivors of out-of-hospital cardiac arrest. Crit. Care Med. 2014, 42, 2401-2408. [CrossRef]

40. Yu, T.; Barbut, D.; Ristagno, G.; Cho, J.H.; Sun, S.; Li, Y.; Weil, M.H.; Tang, W. Survival and neurological outcomes after nasopharyngeal cooling or peripheral vein cold saline infusion initiated during cardiopulmonary resuscitation in a porcine model of prolonged cardiac arrest. Crit. Care Med. 2010, 38, 916-921. [CrossRef]

41. Garrett, J.S.; Studnek, J.R.; Blackwell, T.; Vandeventer, S.; Pearson, D.A.; Heffner, A.C.; Reades, R. The association between intra-arrest therapeutic hypothermia and return of spontaneous circulation among individuals experiencing out of hospital cardiac arrest. Resuscitation 2011, 82, 21-25. [CrossRef]

42. Bernard, S.A.; Gray, T.W.; Buist, M.D.; Jones, B.M.; Silvester, W.; Gutteridge, G.; Smith, K. Treatment of Comatose Survivors of Out-of-Hospital Cardiac Arrest with Induced Hypothermia. N. Engl. J. Med. 2002, 346, 557-563. [CrossRef] [PubMed]

43. Hypothermia after Cardiac Arrest Study Group. Mild therapeutic hypothermia to improve the neurologic outcome after cardiac arrest. N. Engl. J. Med. 2002, 346, 549-556. [CrossRef] [PubMed]

44. Kagawa, E.; Dote, K.; Kato, M.; Sasaki, S.; Oda, N.; Nakano, Y.; Miura, K.; Inoue, I.; Kihara, Y. Do Lower Target Temperatures or Prolonged Cooling Provide Improved Outcomes for Comatose Survivors of Cardiac Arrest Treated with Hypothermia? J. Am. Heart Assoc. 2015, 4, e002123. [CrossRef] [PubMed]

45. Tømte, Ø.; Drægni, T.; Mangschau, A.; Jacobsen, D.; Auestad, B.; Sunde, K. A comparison of intravascular and surface cooling techniques in comatose cardiac arrest survivors. Crit. Care Med. 2011, 39, 443-449. [CrossRef] [PubMed]

46. Pittl, U.; Schratter, A.; Desch, S.; Diosteanu, R.; Lehmann, D.; Demmin, K.; Hörig, J.; Schuler, G.; Klemm, T.; Mende, M.; et al. Invasive versus non-invasive cooling after in- and out-of-hospital cardiac arrest: A randomized trial. Clin. Res. Cardiol. 2013, 102, 607-614. [CrossRef]

47. Nielsen, N.; Wetterslev, J.; Cronberg, T.; Erlinge, D.; Gasche, Y.; Hassager, C.; Horn, J.; Hovdenes, J.; Kjaergaard, J.; Kuiper, M.; et al. Targeted temperature management at $33^{\circ} \mathrm{C}$ vs. $36^{\circ} \mathrm{C}$ after cardiac arrest. N. Engl. J. Med. 2013, 369, 2197-2206. [CrossRef]

48. Bray, J.E.; Stub, D.; Bloom, J.E.; Segan, L.; Mitra, B.; Smith, K.; Finn, J.; Bernard, S. Changing target temperature from $33^{\circ} \mathrm{C}$ to $36{ }^{\circ} \mathrm{C}$ in the ICU management of out-of-hospital cardiac arrest: A before and after study. Resuscitation 2017, 113, 39-43. [CrossRef]

49. Rittenberger, J.C.; Raina, K.; Holm, M.B.; Kim, Y.J.; Callaway, C.W. Association between Cerebral Performance Category, Modified Rankin Scale, and discharge disposition after cardiac arrest. Resuscitation 2011, 82, 1036-1040. [CrossRef] 